\title{
On the Potential of Generic Modeling for VANET Data Aggregation Protocols
}

\author{
Stefan Dietzel*, Frank Kargl*, Geert Heijenk*, and Florian Schaub ${ }^{\dagger}$ \\ *Centre for Telematics and Information Technology, University of Twente, The Netherlands \\ E-mail: \{s.dietzel, f.kargl, geert.heijenk\}@utwente.nl \\ †Institute of Media Informatics, Ulm University, Germany \\ E-mail: florian.schaub@uni-ulm.de
}

\begin{abstract}
In-network data aggregation is a promising communication mechanism to reduce bandwidth requirements of applications in vehicular ad-hoc networks (VANETs). Many aggregation schemes have been proposed, often with varying features. Most aggregation schemes are tailored to specific application scenarios and for specific aggregation operations. Comparative evaluation of different aggregation schemes is therefore difficult. An application centric view of aggregation does also not tap into the potential of cross application aggregation. Generic modeling may help to unlock this potential. We outline a generic modeling approach to enable improved comparability of aggregation schemes and facilitate joint optimization for different applications of aggregation schemes for VANETs. This work outlines the requirements and general concept of a generic modeling approach and identifies open challenges.
\end{abstract}

\section{Motivation}

Vehicular ad-hoc networking (VANET) enables vehicles to communicate with each other on the road. Many applications have been envisioned for this kind of mobile ad-hoc communication. Intersection collision warning, lane merge assistance, smart traffic management, and emergency vehicle warnings are just a few examples of potential applications that promise to enhance road safety, support drivers, or provide infotainment services. Currently, many research projects and field operational trials prepare the deployment of VANET technology in Europe (e.g., CVIS, PRE-DRIVE C2X, simTD), the USA (e.g., VSC, VSC-A), and Japan (e.g., SKY).

A major challenge in the deployment of VANETs is the efficient usage of available bandwidth considering the large number of envisioned applications and the even larger number of potential nodes (vehicles and roadside units). Especially the use of multi-hop dissemination of information that is required by some of the applications creates a huge scalability problem. Thus the development of efficient routing and dissemination protocols has been a major research focus [1]. In-network aggregation is a communication paradigm that has the potential to enhance the scalability of multi-hop communication and, by reducing the required bandwidth per application, enable the coexistence of different applications in the same network. Instead of many nodes sending single messages of similar nature, which are all forwarded individually, data items from multiple messages can be combined into one aggregated message that represents the accumulated content of the single messages.
Consider for example a traffic jam warning application. A vehicle inside a traffic jam detects it by realizing that it is not moving and that its neighbors are also not moving or only moving at slow speed. Without aggregation, the vehicle now sends a warning message reporting the condition and use geobroadcast to disseminate it to vehicles approaching the traffic jam. Other vehicles in the traffic jam will also start generating such warning messages. This traffic information data needs to be disseminated over multiple hops to be useful for car navigation. Instead of forwarding many messages with similar content and, thereby, congesting the wireless medium, vehicles can aggregate their own view of the current situation with warnings received from other vehicles. Such an aggregated message received by a vehicle further away from the traffic jam could contain the information that the traffic jam is 6 kilometers long, its location, and that it contains 312 vehicles traveling at an average speed of $3.2 \mathrm{~km} / \mathrm{h}$.

Thus in-network aggregation has many benefits. Mainly bandwidth requirements can be reduced and less resources are required at receiving nodes, because fewer messages need to be processed and evaluated. Reduced processing and communication requirements also imply reduced energy requirements for on-board units. Moreover, aggregation is inherently privacy friendly, because aggregated information cannot be directly linked to individual vehicles and drivers anymore.

These benefits are well recognized in the VANET research community (cf. Sec. II). But current aggregation schemes and their aggregation functions are often tailored to specific scenarios and information types. As an advantage, these schemes are presumably optimized to their scenarios and applications. The downside is that it is inherently difficult to compare the performance and accuracy of different aggregation schemes. Furthermore, due to missing standards and high specialization, these aggregation schemes cannot support multiple applications simultaneously, thus limiting the overall beneficial impact of aggregation.

We argue that a generic modeling approach for data aggregation in VANETs is required to address these issues and unlock the full potential of in-network aggregation in vehicular networks. Our contribution in this paper is the concept for a modeling framework for aggregation mechanisms that can, in a next step, be used as basis for the design of more generic 
mechanisms. We discuss existing aggregation schemes and their limitations in Section II, and also give an overview of related work on graph-based modeling of spatiotemporal information. Section III outlines our generic modeling approach. Section IV discusses potential benefits of a generic model for evaluation and application independent optimization of data aggregation. Section V outlines open challenges of the generic modeling approach and future work.

\section{RELATED WORK}

In the past years, in-network aggregation schemes for vehicular networks received increasing research attention. The research area is related to aggregation mechanisms for wireless sensor networks (WSNs), but due to differing requirements, like high node mobility in VANETS, WSN aggregation mechanisms cannot be easily adopted [2]. Most VANET aggregation mechanisms are targeted towards one specific use case, often dissemination of average speeds on road segments, while mentioning applicability to other use cases, as well. Similarly, generic modeling schemes for network data have been proposed in different research domains. These models are usually crafted towards centralized systems and used as a data structure to support algorithms working on the contained data.

\section{A. Aggregation Mechanisms}

Wischhof et al. introduced the SOTIS traffic information system [3]. The proposed protocol uses periodic beaconing for exchange of traffic information. Received traffic data is aggregated based on road segmentation. For each segment, the average speed is calculated and later forwarded. Although the authors argue that information precision should decrease with increasing distance, they do not outline how exactly an increase of the segment size depending on distance can be realized in practice.

A more advanced aggregation scheme is applied in the TrafficView system [4]. Similar to SOTIS, it disseminates information about the average speed of vehicles on the road. In contrast to SOTIS, TrafficView is node-centric, not spacecentric. That is, reports of nodes which are close to each other are aggregated by averaging their current speed and position. However, to be able to further identify the nodes, a list of all involved nodes is kept with the aggregate. The aim of this approach is to get an estimated view on the set of surrounding vehicles. To decide the granularity of the aggregation, two algorithms are proposed: a ratio-based and a cost-based. The authors of TrafficView evaluate their system using different metrics that judge the knowledge of a vehicle about its surrounding road network as well as the accuracy of the aggregation.

Lochert et al. [5] take a hierarchical approach on aggregating free parking slots using globally known map data for segmentation. One major advantage of their system is the usage of an adapted version of Flajolet-Martin sketches to achieve a probabilistic but duplicate insensitive sum of free parking spaces. Aggregates can therefore be arbitrarily combined and re-combined without counting free parking slots multiple times.

Van Eenennaam et al. [6] present a system that applies run length encoding to achieve efficient data compression. Instead of averaging information about road segments, only the most relevant single information items for a certain stretch of road are communicated to further away vehicles.

Ibrahim and Weigle [7] present a cluster based aggregation scheme suitable for dissemination of vehicle speeds. Contrary to the previously presented systems, the CASCADE system employs only syntactic, lossless compression of data. At local scope in front of a given vehicle, single reports are disseminated and collected using geo-broadcast. This local view is then clustered using fixed size segments and differential coding is used to compress vehicle information in each cluster. The compressed information is then disseminated further.

Dietzel et al. [2] describe an aggregation scheme that focuses on flexible decision metrics. Fuzzy logic rules are employed to base aggregation decisions on qualitative metrics, such as induced quality loss due to aggregation. The resulting scheme aggregates data more where the road network state is homogenous, yet allots more bandwidth to stretches with high state entropy.

Scheuermann et al. [8] provide a theoretical scalability bound for aggregation protocols in VANETs. The main result is that the data rate must be reduced asymptotically faster than the squared distance to the information source (i.e., $O\left(1 / d^{2}\right)$ ) to be able to scale to larger deployments. Also, the authors provide a construction framework for a mechanism achieving the claimed rate.

\section{B. Graph Modeling}

One common drawback of existing VANET aggregation protocols is their lack of generic mechanisms to model state information. One suitable approach to model such information is based on graphs, due to the fact that road networks themselves are most often modeled as graphs. Therefore, we will shortly present several approaches from the domain of graph modeling, and in particular spatiotemporal graphs, which could be applicable to generalized aggregation schemes.

Ding et al. [9] present a graph model for dynamically changing road networks, including node movements. They provide the definition for what they call a state-based dynamic transportation network (SBDTN). The main goal of the proposed model is to support queries for different network parts at different time instants, thereby supporting routing and planning algorithms. All attributes, including the structure of the road network can change over time. Moreover, the segments of the road network can be in different states, such as free and occupied. However, such states are limited to some discrete values and no explicit support for aggregated data is given.

Similarly, Flinsenberg [10] proposes a graph structure for road network modeling to support route planning algorithms. The information stored in the graph is not the current traffic 


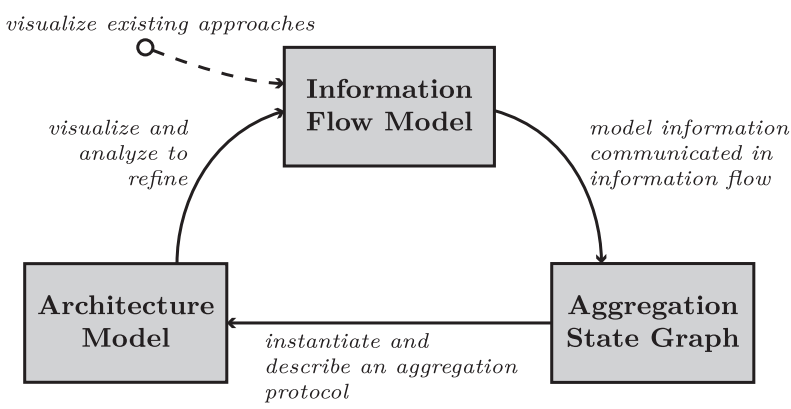

Fig. 1: Aggregation modeling workflow.

state itself, but instead a derived, application specific metric, namely currently reported driving times.

George and Shekhar [11] propose time aggregated graphs as a data model for spatiotemporal networks in general and road networks in particular that supports graph algorithms for shortest path queries. The proposed model is compared to existing approaches that use time expanded networks. The difference is that time extended networks replicate nodes for each instant in time, whereas time aggregated graphs only annotate nodes and edges with the intervals in time during which they are present. Also, the proposed model supports annotating each edge with a number of per-edge-constant values, like travel times.

Kostakos [12] also presents a model for temporal graphs, but uses duplication of nodes per time instance to model temporal connections. Also, weighted edges between time instance of nodes are used to model the time that has passed. However, existing graph modeling approaches are often optimized for use cases, where a central entity has all knowledge about the system. In VANETs, this assumption is not true, because information is distributed throughout the network.

\section{MODELING APPROACH}

The fundamental drawback of existing work is that it addresses either aggregation mechanisms for specific applications in distributed networks or generic model approaches for centralized systems, but not both. Our goal is to propose a modeling framework that takes the generic applicability of graph modeling approaches and applies them to in-network aggregation in VANETs where nodes only have a partial knowledge of the whole network state. Such a model can be used to make existing aggregation schemes more compatible and help the design of suitable comparison metrics. In a next step, the model can be used as the basis for the system design of a generic aggregation scheme. Possibly, the model has to be adapted for the second step, based on the acquired results. The main challenge for the second step is to achieve a system design that is generic enough to support different information items, e.g., traffic state data and weather data, even at the same time. Yet, the system must be efficient enough to be able to disseminate the information with low bandwidth overhead.

We propose a modeling approach comprised of three modeling components (see Figure 1): An information flow model,

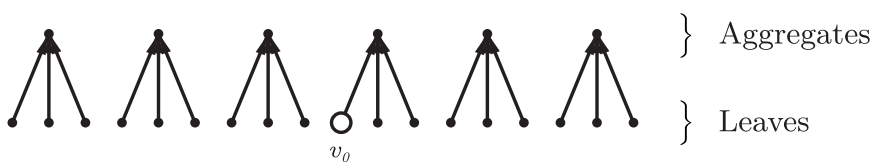

(a) Non-hierarchical fixed-size segments aggregation scheme.

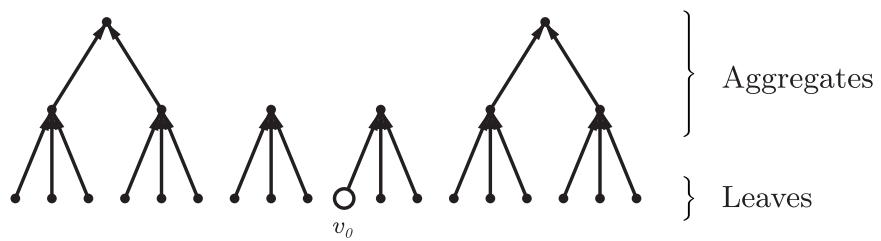

(b) Hierarchical fixed-size segments aggregation scheme with decreasing granularity over distance.

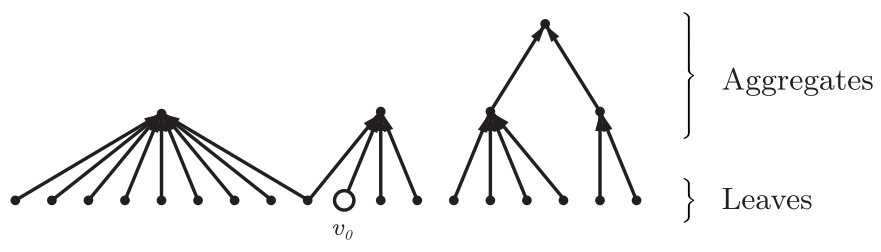

(c) Flexible aggregation scheme.

Fig. 2: Information flow models for various different aggregation schemes.

an aggregation state graph, and an architecture model. The information flow model serves as a visualization tool to understand properties of existing aggregation protocols, and also to exemplify requirements for aggregation mechanisms. The aggregation state graph models the information that is communicated. The goal of the architecture model is to describe all necessary components for the implementation of an aggregation scheme. Finally, the information flow model can be used to validate and analyze the resulting architecture model instantiation. In the following, we will describe our modeling approach and the requirements for each component in detail, and discuss example mechanisms that can be applied to each component.

\section{A. Aggregation Information Flow}

As a first step towards modeling in-network aggregation, we define an information flow model that can be used to understand existing aggregation schemes and model new ones. The goal of the information flow model is to visualize aggregated information and its origins from the viewpoint of one particular vehicle after a given aggregation scheme has run a certain amount of time. To model the information flow, we start with a one-dimensional street, on which a number of vehicles are positioned at regular intervals. All vehicle positions are assumed to be static. Introducing vehicle mobility to the information flow would complicate the resulting graph without providing additional information, because we want to focus on the converged aggregated view of a given road network state. One of the vehicles is the target vehicle, for which we want to visualize the information flow. For each possible target vehicle, the resulting graph would be different, but have the 
same characteristics. The information flow starts as a graph with a single node $v_{0}$, representing the own sensed information (e.g., speed) of the target vehicle. After one protocol step, the target vehicle will have received more information from neighboring vehicles. Consequently, the information flow model is extended by several unconnected nodes, each representing atomic information received from a vehicle. All such leaf nodes are drawn at the position of the corresponding vehicle on the street. Whenever two or more atomic items are aggregated according to the analyzed aggregation scheme, an additional node is added to the graph, one level higher than the leaf nodes. A directed edge is added to the graph from each aggregated leaf node to the node representing the aggregate. Similarly, already aggregated nodes can be aggregated further. Figure 2 shows example information flow models after several protocol steps for different aggregation mechanisms. Note that the actual world model of $v_{0}$ only necessarily contains the topmost nodes of the information flow model. The lower nodes display the history of that information and may or may not be present in addition. The information flow for the target node will eventually converge after several protocol steps. We can use this converged view to analyze a number of characteristics of a given aggregation scheme:

- Support for hierarchical aggregation. If the depth of the resulting graph is 1, an aggregation scheme does not support hierarchical aggregation. Figure 2(a) shows a non-hierarchical aggregation scheme.

- Information dissemination range. The width of the graphs shows the area about which the target node has information. Also, if information is aggregated more with increasing distance to the target node, the graph will represent this by showing higher graph depths with increasing distance to the target node, as seen in Figure 2(b).

- Flexibility of the aggregated view. If all aggregate nodes are connected to the same number of atomic nodes, the scheme is likely to use a fixed threshold for aggregation decisions. Similarly, the more irregular the structure of the information flow model is, the more flexible the underlying aggregation decisions (cf. Figure 2(c)).

Thus the information flow model gives a first idea of how a given aggregation scheme works. While the examples shown are for a one-dimensional street, one can easily extend the model to two-dimensional street networks. The resulting graph structure allows to explore the aggregation decision rules and dissemination scheme of a given aggregation protocol.

\section{B. Aggregation State Graph}

Having a first overview of an aggregation scheme, the next step is to model the information that is communicated by a scheme. Existing aggregation schemes for vehicular networks use a proprietary data representation that is either suited for one particular use case, such as traffic state dissemination, or suited for a class of data, such as traffic state, weather, and available parking spots. Such a proprietary model has two drawbacks. First, it makes it hard to compare two given aggregation schemes when they use different representations of the road network state. Second, such proprietary mechanisms provide no means to support multiple different information items with different data quality requirements at the same time. Therefore we propose to use a generic graph model to represent the network state. At an abstract level, this model serves to transform proprietary data representations of different schemes in one common representation to make them comparable. Moreover, an efficient graph representation, such as adjacency matrices can be utilized to use the state graph as the actual communicated data structure.

The state graph represents three types of information:

- Atomic information. One single information record comprised of sensed information from a single car, containing the current position and time of the car, as well as further information, such as current speed, outside temperature, or a detected free parking spot.

- Aggregated information. Information originating from one or more cars about an interval in time and/or space. Examples for aggregated information are "there are 50 free parking spots in the harbor district", "the average speed on the motorway M1 between kilometer 20 and 25 is $50 \mathrm{kph}$ ", or "on Main Street, at kilometer 6.2, there is an icy road interval of $500 \mathrm{~m}$ length".

- World model. The merged view items of all atomic and aggregated information available to one specific node constitutes the node's world model. The world model can be represented by the state graph, as well. It is the merged view of all atomic and aggregated state graphs available to a particular vehicle at a specific point in time. As vehicles move and information that was priorly unknown is received, the world model is constantly updated.

In general, the network state can be divided into two categories of information. Position or area and time are the dimensions used for indexing the information. Independent of the specific aggregation scheme, this information will always be present. Position is two-dimensional in the generic case, and time is one-dimensional, amounting for a total of three index dimensions. Furthermore, all information we are concerned with originates from vehicles that drive on a defined road network. Therefore, we argue that a graph structure representing the location of information is better-suited than a continuous two-dimensional plane. This graph structure is then annotated with time, as well as any further information that needs to be disseminated for applications, such as speed or temperature. Therefore, the aggregation state graph can leverage on the existing work for graph modeling for spatiotemporal networks, but existing approaches need to be extended to cope with distributed systems and efficiency requirements.

The following are the main additional requirements for network state graphs to apply them to aggregation protocols:

- Fuzzy information. Spatiotemporal road network graphs are usually annotated with specific information for certain parts of the road network. For example, a traffic state graph will contain the current speed per road segment. To deal with aggregated information, graphs need to cope with fuzziness of information. For example, speed could only be available as an average with a given standard deviation. Or the number of 

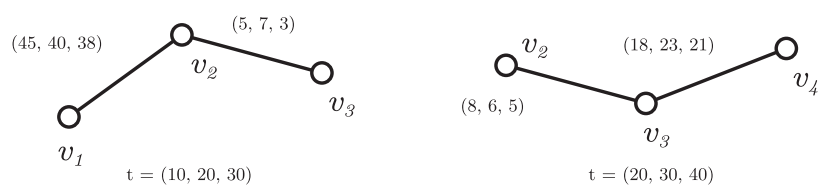

(a) State graphs representing two aggregates with overlapping information.

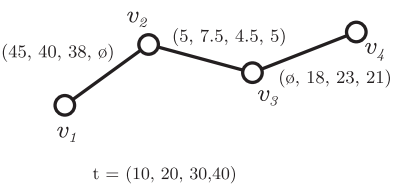

(b) Resulting state graph after fusion of both aggregates.

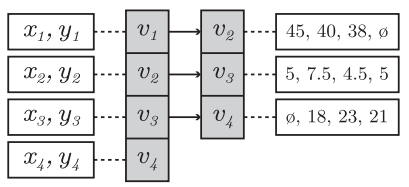

(c) Enhanced adjacency matrix corresponding to the fused graph.
Fig. 3: Aggregation state graph examples.

parking spots could be available as a number plus a certainty in percent, due to imperfect recognition algorithms. Therefore, the aggregation state graph needs to be flexible enough to represent uncertain information in different formats.

- Partial information. Road network graphs for centralized services assume that information about the road structure of the whole network is known. To be suitable for in-network aggregation mechanisms, a state graph needs to be able to cope with partial information. A state graph needs to be able to represent atomic values, as well as aggregated information. Moreover, the graph needs to be flexible enough to represent information about parts of road segments.

- Graph fusion. When previously not known information, represented by a graph, is received by a vehicle, it needs to be comparable to existing graphs representing other partial information. Also, a function needs to be defined that essentially represents the data aggregation mechanism to merge two graph structures into one that represents the aggregated information. This task is easy if the input graphs contain information about disjoint parts of the road network. But if the graphs overlap, conflict resolution mechanisms are required.

- Multiple application domains. Often, graphs represent one particular type of information. However, given the number of different envisioned applications for inter-vehicle networks, it is safe to assume that several different applications requiring a broad set of information types need to be supported at the same time. Due to bandwidth limitations, it is infeasible to run several dissemination protocols in parallel. Therefore, a state graph for in-network aggregation needs to handle more than one type of application data.

- Efficient representation. A graph representation of the network state is already useful per se to compare different aggregation mechanism proposals. However, to achieve a fully generic in-network aggregation scheme, a generic graph structure should also be usable as data exchange format. Therefore, an efficient encoding of the graph representation is needed that can be used to disseminate information in the network.

The following is a simple example graph representation based on [11], assuming that only one data item (average speed) is kept per road segment and that data items remain constant throughout a road segment. Then the state graph can be modeled as $G=\left(V, E,\left(t_{1}, \ldots, t_{k}\right), P, S\right)$, with

- $V=\left\{v_{1}, \ldots, v_{n}\right\}$ the vertices (i.e., intersections and bends) of the road network part modeled by $G$,

- $E=\left\{e_{1}, \ldots, e_{m}\right\}$ the streets connecting the vertices,

- $\left(t_{1}, \ldots, t_{k}\right)$ the time series contained in this state graph,

- $P=\left(p_{1}, \ldots, p_{n}\right)$ the positions of the vertices as GPS coordinates, and

- $S=\left(\left(s_{e_{1}, t_{1}}, \ldots, s_{e_{1}, t_{k}}\right), \ldots,\left(s_{e_{m}, t_{1}}, \ldots s_{e_{m}, t_{k}}\right)\right)$ the average speed driven on a given road segment $e_{i}$ in the time interval $\left[t_{i}, t_{i+1}\right)$.

Figure 3(a) shows two example state graphs for two overlapping parts of the road segment. Figure 3(b) shows the two graphs after fusion. These graphs can be represented by an extended adjacency list, as shown in Figure 3(c). Each node is linked with its position $\left(x_{i}, y_{i}\right)$ (shown on the left) and each link to an adjacent node is annotated with the corresponding measurement series (shown on the right).

However, the adjacency list representation in the example is still too large to be efficiently communicated. Therefore, additional syntactic compression needs to be performed in order to achieve a bandwidth-efficient protocol. Also the graph representation must be extended to support more generic data structures. One evident enhancement is to introduce multiple directed edges between two nodes to represent multiple and directed lanes on each road segment. Furthermore, information that annotates an edge might not be constant for the whole edge. Therefore, either more vertices must be introduced to the graph until each edge only needs to contain constant information. Or edges need to be annotated with polynomial, or even interval-defined functions to represent more complex information about particular segments. However, such generic extensions worsen the efficient encoding problem. Resolving this discrepancy is part of our future work.

\section{Architecture Model}

Finally, the architecture model defines the components of a generic aggregation scheme. Most approaches presented in Section II-A work using an implicit multi-hop dissemination scheme. Instead of directly flooding information into a larger target area, nodes only broadcast current information to their one hop neighbors. To decide on the current information to disseminate, each node keeps all its known information in a world model and selects a certain subset for periodic dissemination. Therefore, relevant information is gradually forwarded and disseminated in a larger region. In addition to the basic dissemination mechanism, two more core components are necessary for aggregation schemes: First, a component that compares new information to already known information and decides whether two items are aggregatable based on some similarity metric. Second, a component that takes several information items and fuses them to a single new information value, possibly inducing information loss. Therefore, we argue that the following four tasks fully describe a generic aggregation scheme: (1) decide whether data items can be aggregated, (2) fuse several data items together, (3) manage the information 


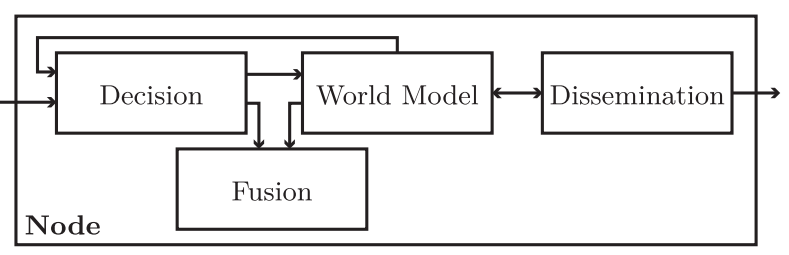

Fig. 4: Generic architecture model.

available to a node in a world model, and (4) disseminate parts of the information to other nodes. As such, the architecture model shows the information flow inside a specific node, whereas the information flow model (Sec. III-A) models the flow of information in the whole network.

Figure 4 shows a high-level architecture view. Information is received, handed to the aggregation decision component and, if possible, to the fusion, and the result is added to the world model. The dissemination component periodically selects a subset of the world model for further dissemination. All information in transit, that is, both aggregated information packets and the world model itself, can be represented by the aggregation state graph, as described in Section III-B.

1) Decision: The decision component is responsible for deciding if two items of information are similar enough to be aggregated. Together with the fusion component, it defines the trade-off of an aggregation scheme. Given two items of information, the decision component needs to decide if they are redundant or similar enough to be aggregated or if they need to be kept separated. To reach the decision, all information contained in the presented items, as well as all information in the world model, can be used. Thus, the aggregation state graph (Section III-B) needs to provide efficient query methods to extract the necessary information.

The simplest form of a decision rule is a threshold decision, which is based on a fixed underlying structure. For example, in [3], items are aggregated if they contain information about the same street segment. However, such a decision is not flexible enough [13], because the amount of information entropy per segment could vary. Moreover, it is not scalable enough [8]. Therefore, the decision component should be able to express a number of influences. These influences should include qualitative aspects, such as quality loss due to aggregation, as well as quantitative aspects, such as street segment or time granularity. More recent proposals include such an explicit trade-off between the loss of data quality and the amount of space saved [4]. That is, given a fixed amount of bandwidth for dissemination, those data items are aggregated where the loss of information is the least. Besides the aforementioned, decision mechanisms such as Bayesian rules, Dempster-Shafer theory, or neuronal networks can be used. Nakamura et al. [14] provide an overview of inference decision methods for WSNs, which can be applied to the decision component for vehicular network aggregation, as well. In particular, fuzzy logic rules, as applied in [2], provide a means to flexibly express aggregation decision rules.
2) Fusion: Once the decision component has selected two items of information for aggregation, the fusion component is in charge of the actual data fusion. In terms of the aggregation state graph, fusion means providing an algorithm to merge two given graphs. Fusion can either be a lossless or a lossy process. One example for lossless fusion of two items would be to keep one value and code the second item as the difference to the first item. However, lossless fusion can be insufficient to achieve the bandwidth reduction required for large-area dissemination of data. Therefore, many existing aggregation mechanisms use lossy fusion of data. Lossy fusion can be as simple as taking the average of two or more values or as elaborate as calculating an approximate, duplicate-insensitive sum using Flajolet-Martin sketches [5]. Also, application of results from audio signal encoding, such as sample and hold signal reconstruction or run-length encoding can be used [15]. In general, lossy fusion schemes require more knowledge about the semantics of the data to be fused. The better the fusion scheme is tailored to a specific use case, the more semantic redundancy can be removed. Therefore, a fusion mechanism cannot be fully generic. Instead, a set of fusion mechanisms needs to be defined, each providing the following desirable properties:

- Hierarchical applicability. Often, support for hierarchical aggregation is necessary, meaning that already aggregated information should be aggregated further. One application for hierarchical aggregation is to provide a rough estimate of the network state far away, while maintaining lesser aggregated version in the closer vicinity. Therefore, fusion functions need to be recursively applicable.

- Duplicate insensitivity. Many events will be sensed by more than one car. Depending on the application domain, it is necessary to filter this duplicate information. Consider a parking spot detection example. If multiple cars sense the same free parking spot, one cannot simply sum up all sensed parking spots, because this would include duplicates. Therefore, duplicate insensitive fusion methods are needed.

- Data quality tracking. One important factor for aggregation decision rules is the data quality loss induced by aggregation. Therefore, it is necessary to keep track of data quality when aggregating. For instance, when data is averaged the standard deviation can be kept as a data quality metric.

An application can then select suitable fusion mechanisms from the given set, or even specify own fusion mechanisms.

3) World model: The world view of a node collects all available information, represented by an aggregation state graph. Note that the information contained in the world model is about certain time intervals, and the world model changes over time, as well when nodes receive information that was priorly unknown. Those two axes of time can correlate, for instance when too old information is purged from the world model. However, the two time axes do not need to correlate, for instance when freshly received information is about older intervals in time. The world model needs to support queries for information about certain regions in an efficient way. The decision component needs to query the world model for po- 
tentially aggregatable information whenever new information is received by the node. The dissemination component also needs to query subsets of the information available in the world model to determine the set of information that needs to be disseminated to neighboring nodes. Abraham et al. [16] provide a survey of suitable index structures. Most importantly, a set of so-called range queries need to be supported, for instance, querying data about a particular part of the road network, or in a certain interval of time.

4) Dissemination: After possible aggregation, the data received by a node needs to be disseminated again. In almost all use cases for aggregation, the dissemination of information in a range larger than one communication hop is necessary. However, it is not necessary to flood information directly over multiple hops. A node can periodically broadcast a subset of its world model to neighboring nodes, which in turn will continue to disseminate the information to nodes further away. Several steps are necessary to select a suitable subset of information for further dissemination. First, a desired bandwidth profile needs to be selected, specifying the average amount of data per time period that can be used for disseminating information. In addition to the global bandwidth limit, also limits per application data type are desirable, according to agreed application priorities.

Next, suitable data needs to be selected for dissemination. A generic selection can include the most recent information from a reasonably large surrounding area. However, some applications will need to define custom selection criteria; therefore, the data selection rules need to be configurable. For instance, a traffic state application might consider information about traffic jams to have higher dissemination priority than information about free flowing traffic. Also, applications might have requirements about the timeliness of message dissemination. In addition to periodic beaconing, more elaborate dissemination algorithms can be used. For instance, carryand-forward can be used by vehicles going in the opposite direction of a traffic jam to inform upcoming vehicles about the congestion.

A generic aggregation scheme needs to support all these criteria. One suitable approach, also applied in earlier work [2], is to apply the idea of relevance-based information dissemination proposed by Kosch et al. [17]. That is, a number of relevance functions can be defined that prioritize the information inside the world model according to different criteria. Multiple of these rating functions can be used in parallel to apply different metrics for different applications. Each of these functions is then assigned a fraction of the total available bandwidth until the limit of the bandwidth profile is reached. This allows a flexible allotment of bandwidth to different applications with different requirements.

5) Summary: Used in the right combination, the decision, fusion, world model, and dissemination components allow for a flexible generic aggregation architecture. The world model and dissemination components provide the basic primitives for implicit multi-hop dissemination. In addition, the decision and fusion components provide the aggregation functionality.

\section{Potential Benefits}

The framework proposed in the previous Section can be used in a two-step process to design a generic aggregation scheme. First, existing schemes can be expressed using the generic framework. This allows to compare the different approaches, and also parts of different approaches to find the most suitable mechanisms. Then, this information can be used to design a generic aggregation mechanism using the proposed framework. The vision is to achieve a generic holistic information dissemination framework that supports different applications with different information quality requirements at the same time.

\section{A. Aggregation Metrics}

The first step towards a generic aggregation scheme is to compare existing schemes and select promising ideas for further development. One drawback of existing work in VANET aggregation schemes is that many different comparison metrics exist, making it hard to compare existing approaches. The information flow model proposed in Section III-A allows a first visual comparison for different schemes. Then, the data format used by the different schemes can be generalized using an aggregation state graph as described in Section III-B. If the state graph representation is generic enough to express all schemes, this provides a common view on the data quality achieved by existing schemes. Finally, the architecture model (Section III-C) allows to disassemble the different approaches into their components. This highlights the focus of the different schemes and allows to take parts of existing schemes, extract their underlying ideas, and apply them when designing a generic aggregation scheme.

\section{B. Generic Aggregation Scheme}

Having compared and thoroughly assessed existing schemes, the next step is to combine different existing approaches and find new ones to instantiate a generic aggregation mechanism. The main challenges here are (1) to find an aggregation state graph representation that is generic enough, yet still efficiently encodable to be disseminated throughout the network and (2) to instantiate the generic architecture model components with concrete mechanisms.

The goal of the generic framework is to support a wide range of possible application data and also multiple types of application data at the same time. In a first step, one can assume that different applications with the same quality requirements use the aggregation mechanism at the same time. This assumption reduces the problem of conflict resolving between different application requirements. The advantage of such a generic scheme over existing schemes is that it allows for a clear definition of quality requirements and bandwidth profiles. Then, all kinds of applications, such as traffic state dissemination, parking spot availability, or critical road condition warnings, can use the same dissemination mechanism, having clear assertions of the level of data quality it will provide and the level of bandwidth it will consume. 


\section{Vision of a Holistic Information Distribution Framework}

Having designed a generic aggregation scheme, the ultimate vision is to have a holistic dissemination framework for all kinds of traffic application data. All applications that need to disseminate data in the network can then specify their level of data quality, desired granularity, as well as target dissemination area and dissemination frequency. Likewise, a total amount of available bandwidth and a priority ranking of different applications is agreed upon. The generic aggregation mechanism should then be able to flexibly adopt to the given requirements and configure fusion and dissemination mechanisms accordingly. The main challenge in contrast to a generic aggregation mechanism that supports one global quality requirement is to be able to handle conflicting application data requirements. One possible approach is to apply the idea of progressive data encoding found in image compression algorithms, such as JPEG. Given a set of applications, first a minimum required data quality is derived. Then, information of that quality can be disseminated periodically. In addition, more fine granular data is disseminated for applications that require it. This additional data can be encoded as the differences between the baseline coarse data and the more exact data, combining lossy and lossless compression techniques.

Moreover, depending on the desired dissemination range and requirements on dissemination frequency, a fully generic dissemination scheme could dynamically decide on the communication channel to use. While more timely data about the closer vicinity is disseminated via vehicle-to-vehicle communication, less timely information for larger target regions could be disseminated using cellular network broadcasts, or peer to peer overlays over cellular networks, such as proposed in [18]. While this vision is far from reality today, the basic ingredients exist and it would allow a wide range of envisioned VANET applications to coexist in a bandwidth-efficient manner.

\section{COnClusion And Future Work}

In this paper, we presented models for generic aggregation in VANETs. We presented selected existing research on VANET aggregation protocols and graph modeling approaches for traffic information systems and other domains. Both research directions are interesting, but must be combined and extended to achieve a truly generic distributed information aggregation and dissemination scheme. In this paper we have outlined a modeling framework that can be used to extract information about existing schemes, make them comparable, and facilitates the design of a generic aggregation scheme. For each component, we have outlined the desired functionality, as well as specific requirements, open challenges, suitable examples from related work, and new ideas. The detailed specification of each modeling component is still not concluded and needs the combination of work and expertise from different research fields to arrive at a versatile and suitable model. Especially the development of a generic aggregation state representation is an open challenge, yet it is needed to serve as the data structure for a generic aggregation scheme.
While it has not been demonstrated yet that a generic aggregation scheme can really be constructed, we consider it necessary to generalize and unify existing approaches. If in a future VANET deployment each application runs a separate aggregation scheme, then benefits of aggregation will be negated soon. Currently, we are working on further detailing especially generic state graphs suitable for aggregation, but we are also refining the architecture model to apply it to different existing aggregation schemes as a comparison metric.

\section{REFERENCES}

[1] E. Schoch, F. Kargl, M. Weber, and T. Leinmuller, "Communication patterns in vanets," Communications Magazine, IEEE, vol. 46, no. 11, pp. $119-125$, nov. 2008.

[2] S. Dietzel, B. Bako, E. Schoch, and F. Kargl, "A fuzzy logic based approach for structure-free aggregation in vehicular ad-hoc networks," in Proceedings of the sixth ACM international workshop on VehiculAr InterNETworking. New York,USA: ACM Press, 2009, pp. 79-88.

[3] L. Wischhof, A. Ebner, H. Rohling, M. Lott, and R. Halfmann, "SOTIS - a self-organizing traffic information system," in The 57th IEEE Semiannual Vehicular Technology Conference, 2003. Ieee, 2003, pp. 2442-2446.

[4] T. Nadeem, S. Dashtinezhad, C. Liao, and L. Iftode, "TrafficView: traffic data dissemination using car-to-car communication," ACM SIGMOBILE Mob. Comp. and Comm. Review, vol. 8, no. 3, p. 6, Juli 2004.

[5] C. Lochert, B. Scheuermann, and M. Mauve, "Probabilistic aggregation for data dissemination in VANETs," in Proceedings of the fourth ACM international workshop on Vehicular ad hoc networks. New York, New York, USA: ACM Press, 2007, pp. 1-8.

[6] M. van Eenennaam and G. Heijenk, "Providing Over-the-horizon Awareness to Driver Support Systems," in Proceedings of 4th IEEE Workshop on Vehicle to Vehicle Communications, 2008.

[7] K. Ibrahim and M. C. Weigle, "CASCADE: Cluster-Based Accurate Syntactic Compression of Aggregated Data in VANETs," in 2008 IEEE Globecom Workshops. IEEE, November 2008, pp. 1-10.

[8] B. Scheuermann, C. Lochert, J. Rybicki, and M. Mauve, "A fundamental scalability criterion for data aggregation in VANETs," in Proceedings of the 15th annual international conference on Mobile computing and networking. New York, New York, USA: ACM Press, 2009, p. 285.

[9] Z. Ding and R. Güting, "Modeling temporally variable transportation networks," in Database Systems for Advanced Applications. Springer, 2004, pp. 651-724.

[10] I. C. Flinsenberg, "Route Planning Algorithms for Car Navigation," PhD Thesis, Technische Universiteit Eindhoven, 2004.

[11] B. George and S. Shekhar, "Time-aggregated graphs for modeling spatiotemporal networks," in Journal on Data Semantics XI, ser. Lecture Notes in Computer Science, S. Spaccapietra, J. Pan, P. Thiran, T. Halpin, S. Staab, V. Svatek, P. Shvaiko, and J. Roddick, Eds. Springer Berlin / Heidelberg, 2008, vol. 5383, pp. 191-212.

[12] V. Kostakos, "Temporal graphs," Physica A: Statistical Mechanics and its Applications, vol. 388, no. 6, pp. 1007-1023, Mar. 2009.

[13] S. Dietzel, E. Schoch, B. Bako, and F. Kargl, "A Structure-free Aggregation Framework for Vehicular Ad Hoc Networks," in Proceedings of the 6th International Workshop on Intelligent Transportation, Hamburg, Germany, 2009, pp. 61-66.

[14] E. F. Nakamura, A. A. F. Loureiro, and A. C. Frery, "Information fusion for wireless sensor networks: Methods, models, and classifications," ACM Comput. Surv., vol. 39, no. 3, p. 9, 2007.

[15] M. van Eenennaam and G. Heijenk, "Providing over-the-horizon awareness to driver support systems," in Fourth International Workshop on Vehicle-to-Vehicle Communications. Enschede: University of Twente, June 2008, pp. 19-25.

[16] T. Abraham and J. F. Roddick, "Survey of spatio-temporal databases," GeoInformatica, vol. 3, pp. 61-99, 1999, 10.1023/A:1009800916313.

[17] T. Kosch, C. J. Adler, S. Eichler, C. Schroth, and M. Strassberger, "The scalability problem of vehicular ad hoc networks and how to solve it," IEEE Wireless Communications, vol. 13, no. 5, pp. 22-28, October 2006.

[18] J. Rybicki, B. Scheuermann, M. Koegel, and M. Mauve, "PeerTIS: a peer-to-peer traffic information system," in Proceedings of the sixth ACM international workshop on VehiculAr InterNETworking. New York, New York, USA: ACM Press, 2009, p. 23. 